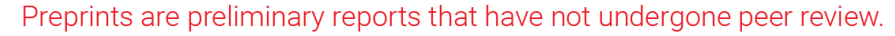 They should not be considered conclusive, used to inform clinical practice, or referenced by the media as validated information. \\ Game Theory-Based Power Allocation Strategy for NOMA in 5G Cooperative Beamforming
}

\section{Mohammed Fadhil}

Universiti Kebangsaan Malaysia Faculty of Engineering and Built Environment

\section{Anabi Hilary Kelechi}

Air Force Institute of Technology

Rosdiadee Nordin ( $\square$ adee@ukm.edu.my )

Universiti Kebangsaan Malaysia https://orcid.org/0000-0001-9254-2023

\section{Nor Fadzilah Abdullah}

Universiti Kebangsaan Malaysia Faculty of Engineering and Built Environment

\section{Mahamod Ismail}

Universiti Kebangsaan Malaysia Faculty of Engineering and Built Environment

\section{Research Article}

Keywords: Non-orthogonal multiple access (NOMA), cooperative beamforming, nash bargaining solution.

Posted Date: July 20th, 2021

DOI: https://doi.org/10.21203/rs.3.rs-398467/v1

License: (c) (i) This work is licensed under a Creative Commons Attribution 4.0 International License. Read Full License

Version of Record: A version of this preprint was published at Wireless Personal Communications on August 18th, 2021. See the published version at https://doi.org/10.1007/s11277-021-08941-y. 


\title{
Game Theory-Based Power Allocation Strategy for NOMA in 5G Cooperative Beamforming
}

\author{
Mohammed Fadhil ${ }^{1}$, Anabi Hilary Kelechi ${ }^{2}$, Rosdiadee Nordin ${ }^{1}$, Nor Fadzilah Abdullah ${ }^{1}$ and Mahamod Ismail ${ }^{1}$ \\ ${ }^{1}$ Department of Electrical, Electronic \& Systems Engineering, Faculty of Engineering and Built Environment, \\ Universiti Kebangsaan Malaysia, 43600 UKM Bangi, Selangor, Malaysia \\ ${ }^{2}$ Department of Aerospace Engineering, Air Force Institute of Technology, Kaduna, Nigeria \\ mohammedfadhilm@gmail.com, hk.anabi@afit.edu.ng, adee@ukm.edu.my, fadzilah.abdullah@ukm.edu.my, \\ andmahamod@ukm.edu.my \\ Corresponding Author: Rosdiadee Nordin
}

\begin{abstract}
Non-orthogonal multiple access (NOMA) is a Fifth Generation (5G) technique that allows many users to simultaneously access the same time-frequency separating channels via successive interference cancellation (SIC) receiver. Cooperative NOMA (CNOMA) is an effective tool to prevent performance degradation of far users by allocating minimal power to users with good channel conditions. In this paper, we proposed a fair power and channel allocation scheme based on the Nash bargaining solution (NBS) game solution in full-duplex, cooperative beamforming (BF) for multicarrier (MC) NOMA. The proposed NBS scheme assigns optimal power and channel allocation according to channel conditions while maintaining a fair rate amongst cooperative users. NBS provides a fair and optimum approach for maximizing the total rate of CNOMA. The signal-to-leakage (SLR) ratio precoding technique is considered as a design performance criterion for beamforming vector towards achieving power domain CNOMA players. Simulation results show that at $B E R=10^{-5}$, the NBS power allocation (proposed scheme) improved by $2 \mathrm{~dB}$ in terms of Signal-toNoise Ratio (SNR), compared with the non-cooperative scheme, and $3 \mathrm{~dB}$ compared with the multiple-input multiple-output NOMA (MIMO-NOMA). In terms of fairness, the proposed NBS scheme shown a high level of fairness at 0.8401 , compared to the other similar approaches in the literature.
\end{abstract}

Index Terms - Non-orthogonal multiple access (NOMA), cooperative beamforming, nash bargaining solution.

\section{INTRODUCTION}

NON-orthogonal multiple access (NOMA) has recently received extensive attention compared with conventional orthogonal multiple access because it allows multiple users to communicate with each other simultaneously using the same time/frequency channel, leading to enhanced spectral efficiency [1]. Successive interference cancellation (SIC) is applied at users' receivers to separate the superposition of different power levels of users' signals [2][3]. NOMA with cooperation (CNOMA) between superposition users' signals is a candidate solution proposed to prevent performance degradation of far users [4][5][6]. Hence, the best users channel can exploit redundant information by acting as relays to improve the reliability of other users who have a poor connection with BS. Most studies on CNOMA assumed that the communication channel between the base station (BS) and users adopts a Rayleigh fading 
channel, which is characterized by Non line of sight (NLOS) signal scattering scenario. This assumption is short sighted based on the premise that both line of sight (LOS) and NLOS are expected to feature predominantly in the emerging scenario.

In 5G applications, such as massive Internet of things (IoT), and machine-type communications low-cost sensors required a small area, which can be better exhibited by the Rician fading channel because both LOS and NLOS exist. Exploiting the emergence of LOS and NLOS loophole, the performance of the NOMA scheme was evaluated with Rician fading channels and noticeable results observed [7]. NOMA technology can be divided into: (i) power domain multiplexing and (ii) code domain multiplexing [8-10]. Power domain multiplexing is quite challenging because of the need to execute an optimal power allocation scheme, which is important for the NOMA systems' overall performance.

Many studies on resource allocation have included power allocation and proposed optimization methods. For example, [11] jointly optimised power and subcarrier allocation for multicarrier NOMA (MC-NOMA). MC-NOMA is NP-hard and solved using the Lagrangian dual optimisation and dynamic programming technique. In [12], an algorithm for subchannel assignment and power allocation across subchannels was proposed to maximize the energy efficiency in MC-NOMA systems. In [13], maximization of the total sum rate by fairness between users was performed for optimal power allocation, and subchannel assignment in MC-NOMA was proposed. The author in [14] proposed a resource allocation algorithm for full-duplex MC-NOMA systems to maximise the weighted sum throughput of the system. Other studies have proposed the use of beamforming with NOMA. For example, [15] aimed to minimise transmission power depending on the beamforming design in multiple-input multiple-output (MIMO)-NOMA.

Driven by the beamforming vector design concept, two NOMA concepts have been established in the literature, which is: clustering NOMA [15] and non-clustering NOMA [16], [17]. In the clustering NOMA scheme, the users are grouped into many clusters (at minimum two users in each cluster). Consequently, each transmits beamforming vector is assigned to support one cluster. In the non-clustering NOMA scheme, there is no clustering assigned and each user is supported by its own beamforming vector. In fact, clustering is used to support a huge number of users to reduce the separation complexity at SIC. In [18], beamforming vectors were employed in the multiuser transmitted system, and each user was assigned a single antenna. The proposed scheme aimed to guarantee user fairness and used channel gain as a constraint factor. Among the available methods for power allocation, the Nash bargaining solution (NBS) game theory has been suggested.

Game theory has been chosen to achieve better payoffs by cooperation between users to share some information. Players can determine whether there is a potential extra utility for everyone if they cooperate. If there is such extra utility, players may bargain with each other to decide how to share information. Thus, without losing the generality of the NBS, our contributions are:

1. We propose NBS as a method for power allocation in non-clustering full-duplex, cooperative beamforming (BF) for multicarrier non-orthogonal multiple access NOMA.

2. Mathematical model for implementing a fair NBS scheme for optimal power allocation cooperative BF for MC-NOMA system. The proposed scheme assigns optimal power and 
channel allocation according to channel conditions, while keeping a fair rate amongst cooperative users.

3. The performance of the proposed NBS-based optimal power allocation scheme is validated and compared against the other scheme in the literature based on BER performance and fairness gain.

The limitation of employed NBS in any system is the requirement of convex utility space. In the application of a multiuser communication system, the information rate is chosen as a user utility. The interference amongst the users will push the utility space (rate region) from a convex to the non-convex domain. Orthogonal signalling, such as frequency division multiple access and time division multiple access, converts the non-convex utility space to a convex one, which is considered a drawback of using NBS [19] with NOMA. Thus, in this study, we adopt signal-to-leakage ratio (SLR) [20-22] as a beamforming vector in order to:

1. To reduce the interference amongst users' which leads to maintaining the convex utility space of NBS. Since maximizing the value of SLR is expected to improve the desired user's power level and reduces the interference to other users from the desired user.

2. As a technique to circumvent the coupled variables problem, we suggest SLR as an optimization criterion in the achievable rate equation, instead of using SINR, which leads to the coupled variables problem.

Meanwhile, NBS offers a fair and optimum approach to maximize the total rate of the CNOMA system. Our proposed power allocation game scheme considers the Rayleigh fading channel as a communication channel between the BS and each user (first-time slot), whereas Rician fading channels consider as inter-user channels between users (second-time slot). The reason for chosen Rician fading channels in the second time slot is simply because there is LOS between a user and the next user. Hence, the need for cooperation.

The remainder of this paper is organized as follows. Section II introduces the system model of downlink BF for MC of CNOMA (the proposed scheme), the channel model description (Raleigh and Rician Fading), and the employing of SIC in a cooperative scheme. Section III presents the problem formulation include the SLR beamforming analysis, interference analysis, and SLR limit theorem. In Section IV, the bargaining solution has been addressed starting with power allocation based on NBS, which involved optimization problems, the existence of NBS, and the NBS Scheme for power allocation. According to the studies in literature, the best performance of NOMA are shown when two users are considered, therefore the proposed scheme validated with the previous two users MIMO-NOMA [23] study in terms of bit error rate while the fairness performance of the proposed scheme validated with the previous study in term of the Jain's fairness index. The conclusions are provided in Section IV.

\section{SYSTEM MODEL}

The downlink of the full-duplex cooperative BF for the MC-NOMA system consists of $U$ cooperative users and one BS. Each of the cooperative users is assumed to be equipped with $N_{U}$ antennas, and the BS is assumed to be equipped with $M$ antennas. The proposed scheme considers beamforming based maximal SLR in MC-CNOMA to achieve power domain superposition cooperation in users' signals. More explicitly, SLR beamforming vectors is 
suggested as an optimization criterion in achievable rate equation instead of using SINR, since using SINR as optimization criterion will lead to coupled variables problem. The block diagram of the downlink full-duplex cooperative BF MC- NOMA is shown in Fig. 1.

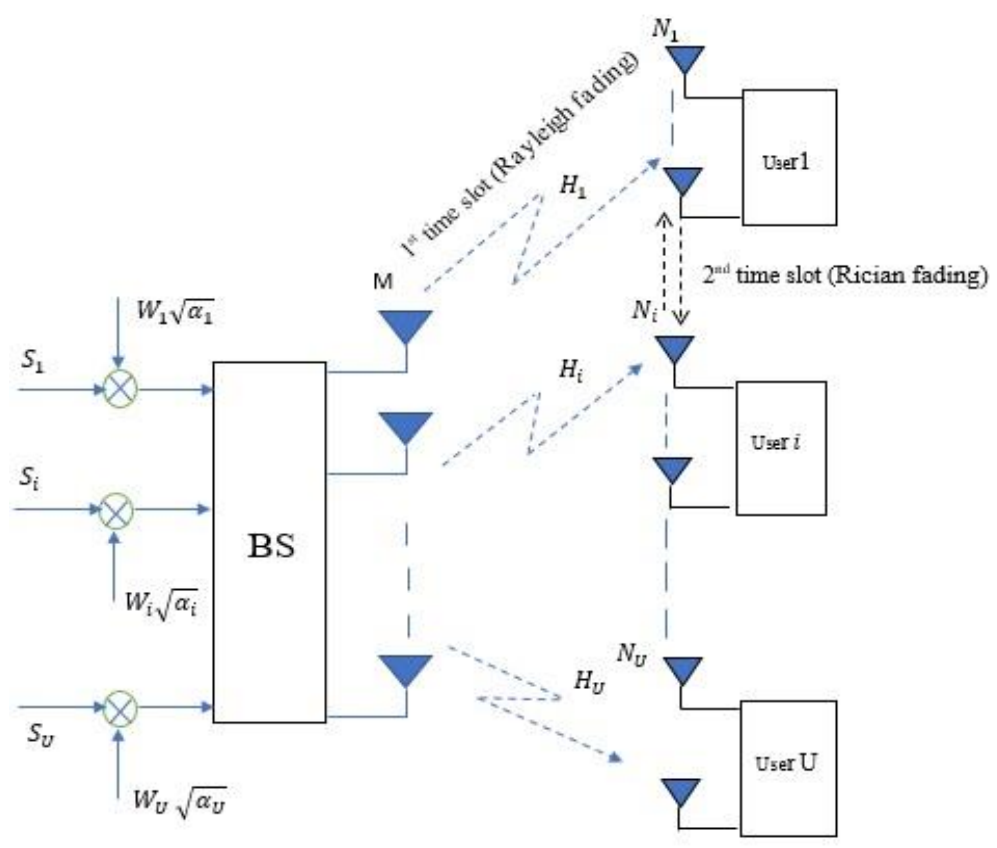

Fig. 1. Simplified full-duplex cooperative BF for MC of NOMA for downlink.

Total bandwidth $B$ is divided into $C$ subchannels, each of which has bandwidth $\frac{B}{C}$. There are two techniques to assign subchannel in NOMA. The first technique considers that each user can use all the available subchannels by sharing the same time and frequency and exploiting the difference in power levels. Meanwhile, the second technique considers that each user can use one subchannel or more that is similar to OFDM but allows the exploitation of different power levels instead of exploiting the orthogonality between subcarrier signals. The concept applies both uplink and downlink transmission [24]. We define $\alpha_{i, c}$ as the power allocated to user $i, i \in\{1, \ldots, U\}$ on subcarrier $c$. In the resource allocation problem, we use parameter $i$ to denote the set of users allocated with positive power $\alpha_{i, c} \geq 0$ to subchannel $c$ [25].

\section{A. Assumptions}

i) The transmitted symbols of the $i^{\text {th }}$ desired user is $s_{i}$ and the $u^{\text {th }}$ interfering symbols $s_{u}$ are assumed to be zero-mean and unit variance.

ii) The antenna spacing at the receiver is sufficiently large so that the fading at each antenna is spatially uncorrelated, i.e., the channel vector $H_{i}$ is distributed as $\mathbb{C N}(0,1)$. Further, the interfering channels are also spatially uncorrelated, implying that the $i^{\text {th }}$ interfering vector $H_{i}$ is distributed as $\mathbb{C N}(0,1)$.

iii)The fading coefficient vectors, and the noise vector $n$ are uncorrelated.

\section{B. Raleigh Fading Channel Model}


During the first time slot of the CNOMA system, the BS transmits a superposition of the individual messages on subchannel $c$, i.e, $w_{i} s_{i}$, to all users over Rayleigh fading channels. $s_{i}$ and $w_{i}$ are symbols intended for the $i^{t h}$ user and the corresponding beamforming weight, respectively, as shown in Fig. 1. NBS is applied to allocate optimal power $\alpha_{i}$ and implement channel assignment to each user before user cooperation. The received signal at the $i^{\text {th }}$ user is given by [20]:

$$
y_{i}=H_{i} w_{i} \sqrt{\alpha_{i}} s_{i}+H_{i} \sum_{u=1, u \neq i}^{U} w_{u} \sqrt{\alpha_{u}} s_{u}+n_{i}
$$

where $s_{i}$ denotes the transmitted data intended for user $i$. The scalar data $s_{i}$ is multiplied by an $M \times 1$ beamforming vector $w_{i}$ before being transmitted over the channel, $\alpha_{i}$ is the power allocation factor for strong user $i, n_{i}$ is an additive white Gaussian noise (AWGN) vector whose entries are independent and identically distributed (i.i.d.) with zero mean and variance $\sigma_{i}^{2}, w_{u} s_{u}$ is the co-channel interference (CCI) caused by the multi-user nature of the system. and $H_{i}$ is given as [20]:

$$
H_{i}=\left[\begin{array}{ccc}
h_{i}^{(1,1)} & \ldots & h_{i}^{(1, M)} \\
\vdots & \ddots & \vdots \\
h_{i}^{\left(N_{u}, 1\right)} & \ldots & h_{i}^{\left(N_{u}, M\right)}
\end{array}\right]
$$

where $h_{i}^{(n, m)}$ represents the channel coefficient that affects the propagation signal between the $m^{\text {th }}$ transmitter array antenna of BS and the $n^{\text {th }}$ receiver array antenna of the $i^{\text {th }}$ user.

\section{Rician Fading Channel Model}

SIC is used at the receiver of each user by exploiting maximal SLR and NBS to provide an optimal power domain for transmitting multiple signals over the same frequency and time domain. To achieve power and channel allocation, cooperative users should be ordered based on their channel quality from the BS [16][17], i.e., $\left\|h_{1}\right\|_{2} \leq\left\|h_{2}\right\|_{2} \leq\left\|h_{U}\right\|_{2}$.

During the second time slot, following this previous order, the SIC in the full-duplex cooperative user's strategy will detect each user as follow: user $i$ detects the first $i-1$ users' signals by using SIC and sends the $i-1$ users' signals to the user over a Rician fading channel. Meanwhile, user $i-1$ detects its signal by using SIC and sends $i$ users' signals to another user during the second time slot over a Rician fading channel. In the same way, the message of other users, i.e., from $i+1$ to $U$, is sent to user $i$ in the second time slot over a Rician fading channel. In other words, the $i^{\text {th }}$ user's signal should be detected by user $l$ for all $l \in\{i, i+1, \ldots, U\}$. This study focuses on optimal power allocation and channel assignment based on NBS to maximize the total rate of cooperative BF of MC-NOMA. Hence, the remaining signal at $l$ to detect the $i^{\text {th }}$ user is presented as follows:

$$
y_{i}^{l}=H_{l} w_{i} \sqrt{\alpha_{i}} s_{i}+H_{l} \sum_{u=i+1}^{U} w_{u} \sqrt{\alpha_{u}} s_{u}+n_{i}
$$

Specifically, users with good channel conditions have prior information on the messages of 
other users, and users with poor channel conditions have information on other users, including those with good channel conditions.

\section{Cooperative Communication}

For a thorough understanding of full-duplex cooperative BF for MC-NOMA, we considered two cooperative users $i^{\text {th }}$ and $(\mathrm{i}+1)^{\text {th }}$ in the beamforming downlink scheme, as shown in Fig. 2. In the NOMA full-duplex cooperative BF strategy, both users act as a relay and exploit redundant information for other users to improve their reliability and prevent the degradation of users who have a weak connection with BS. The superposition information is transmitted in two time slots, namely, direct and cooperative phases.

In the direct transmission phase, a superposed message of users $i$ and $i+1\left(\sqrt{\alpha_{i}} s_{i}+\right.$ $\sqrt{\alpha_{i+1}} s_{i+1}$ ) [6], is transmitted by BS. Assuming that the channel condition of user $i+1$ is the better channel condition, the SIC technique is employed in both users' receiver. Thus, user $i+1$ decodes the information of user $i$ before decoding its own information. During the second time slot, user $i+1$ starts working as a relay and forwards the prior decoded information $\sqrt{\alpha_{\mathrm{i}}} s_{i}$ of user $i$. Meanwhile, user $i$ decodes its own information then decodes user $i+1$ 's information. During the second time slot, user $i$ starts working as a relay and forwards the information $\sqrt{\alpha_{i+1}} s_{i+1}$ of user $i+1$.

Therefore, two copies of signals are received by each user through different paths. The reliability of signal reception of the user with poor channel conditions is improved by having two copies of the message. The strong user channel is also enhanced by having two copies. The channel model of full-duplex cooperative BF for MC of NOMA is shown in Fig. 2.

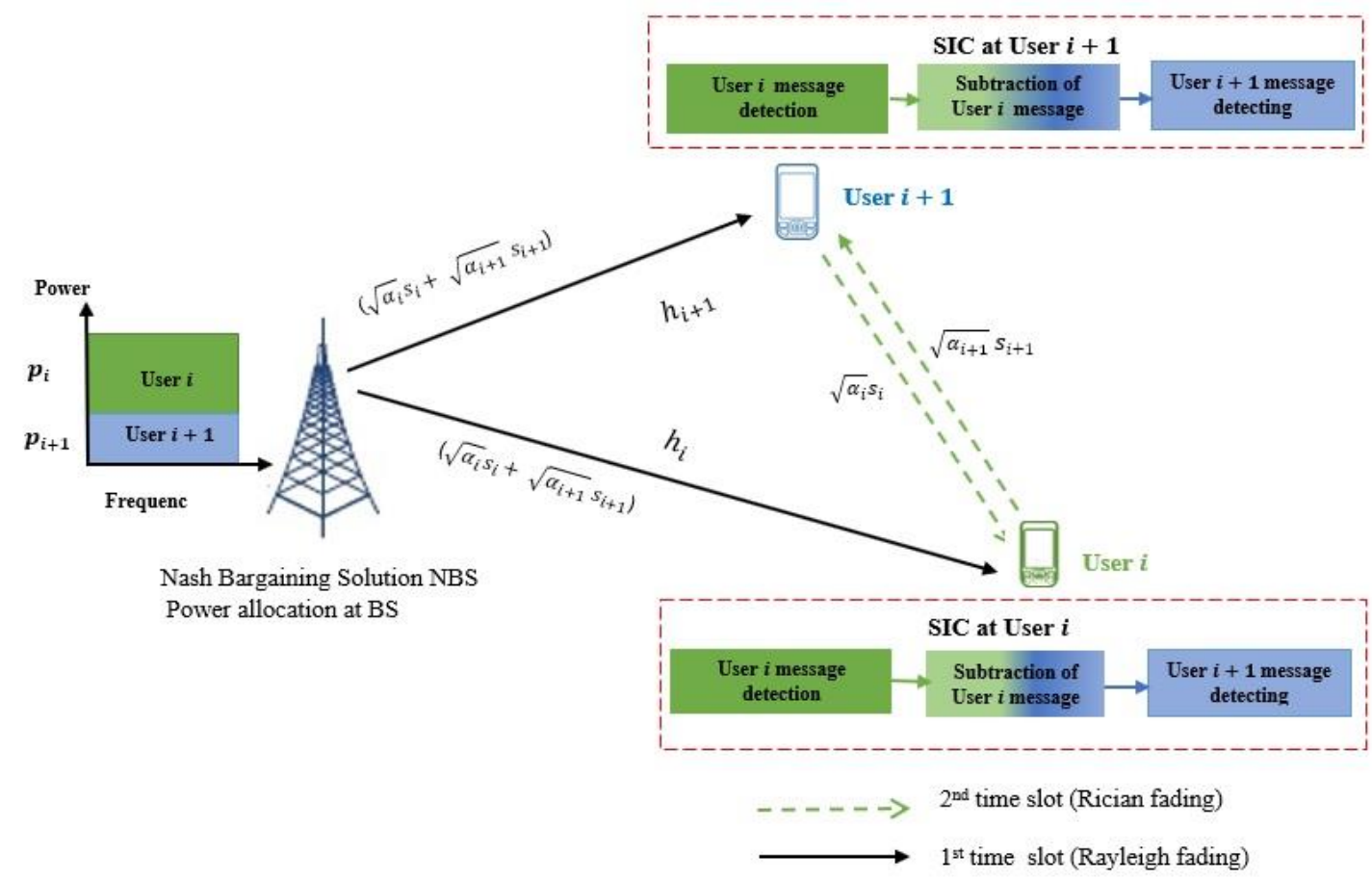


Fig. 2. Two-users NBS power allocation in full-duplex cooperative BF for MC of NOMA channel model.

The maximum ratio combiner (MRC) receiver is considered, since MRC has lesser complexity and achieves the best performance comparing with another estimators (i.e zero forcing ZF estimator) [20], especially when employed MRC with SLR beamforming techniques and SIC technique. For user $i$, the MRC detection scheme is used to estimate $s_{i}$ signal from the received signal as follows [20]:

Then,

$$
\tilde{y}_{i}=\frac{w_{i}^{*} H_{i}^{*}}{\left\|H_{i} w_{i}\right\|^{2}} y_{i}
$$

$$
\tilde{y}_{i}=\sqrt{\alpha_{i}} s_{i}+\frac{w_{i}^{*} H_{i}^{*} \sum_{u=1 . u \neq i}^{U} \sqrt{\alpha_{u}} H_{i} w_{u} s_{u}}{\left\|H_{i} w_{i}\right\|^{2}}+\frac{w_{i}^{*} H_{i}^{*}}{\left\|H_{i} w_{i}\right\|^{2}} n_{i}
$$

In the transmitter of the proposed scheme, the constraint of transmission power is employed and described as $\left(\left\|\beta_{i} s_{i}\right\|^{2}\right) \leq P_{i}$, where $\beta_{i}$ is a constant to meet the total transmitted power constraint, and it is given as [26].

$$
\beta_{i}=\sqrt{\frac{N_{T}}{\operatorname{Tr}\left(H^{-1}\left(H^{-1}\right)^{H}\right)}}
$$

According to [26], the received symbol $s$ is preceded by pre-equalization weight $\mathrm{w}$, so $\widehat{s}=$ $w s$, where $w=\beta H^{-1}$. Therefore, the transmitted signal to the $i^{\text {th }}$ user in the second time slot is

$$
\hat{s}_{u-i-2 n d}=w s_{u-i-1 s t}
$$

where $s_{u-i-1 s t}$ is the leakage signal from the $i^{t h}$ user detected by the $u^{\text {th }}$ user in the first time slot. The received signal in the second time slot by $i^{\text {th }}$ user is given by

$$
y_{u-i-2 n d}=H_{u-i-2 n d} \hat{s}_{u-i-2 n d}+n_{i}
$$

where $H_{u-i-2 n d}$ represents the inter-user channel between $u^{\text {th }}$ and $i^{\text {th }}$ users and $n_{i}$ is the AWGN in the $i^{\text {th }}$ user. The MRC is used to combine the desired signal $\widehat{s}_{i}$ (which is detected by itself as its own signal in the first time slot) with $\hat{s}_{u-i-2 n d}$ (leakage signal detected by the second user in the second time slot), as shown in Fig. 3.

$$
\begin{array}{r}
s_{i}=\widehat{s}_{i}+\sum_{u=1, u \neq i}^{U} \hat{s}_{u-i-2 n d} \\
s_{i}=\widehat{s}_{i}+\sum_{u=1, \quad u \neq i}^{U} \frac{H_{u-i-2 n d}^{*}}{\left\|H_{u-i-2 n d}\right\|^{2}} y_{u-i-2 n d}
\end{array}
$$




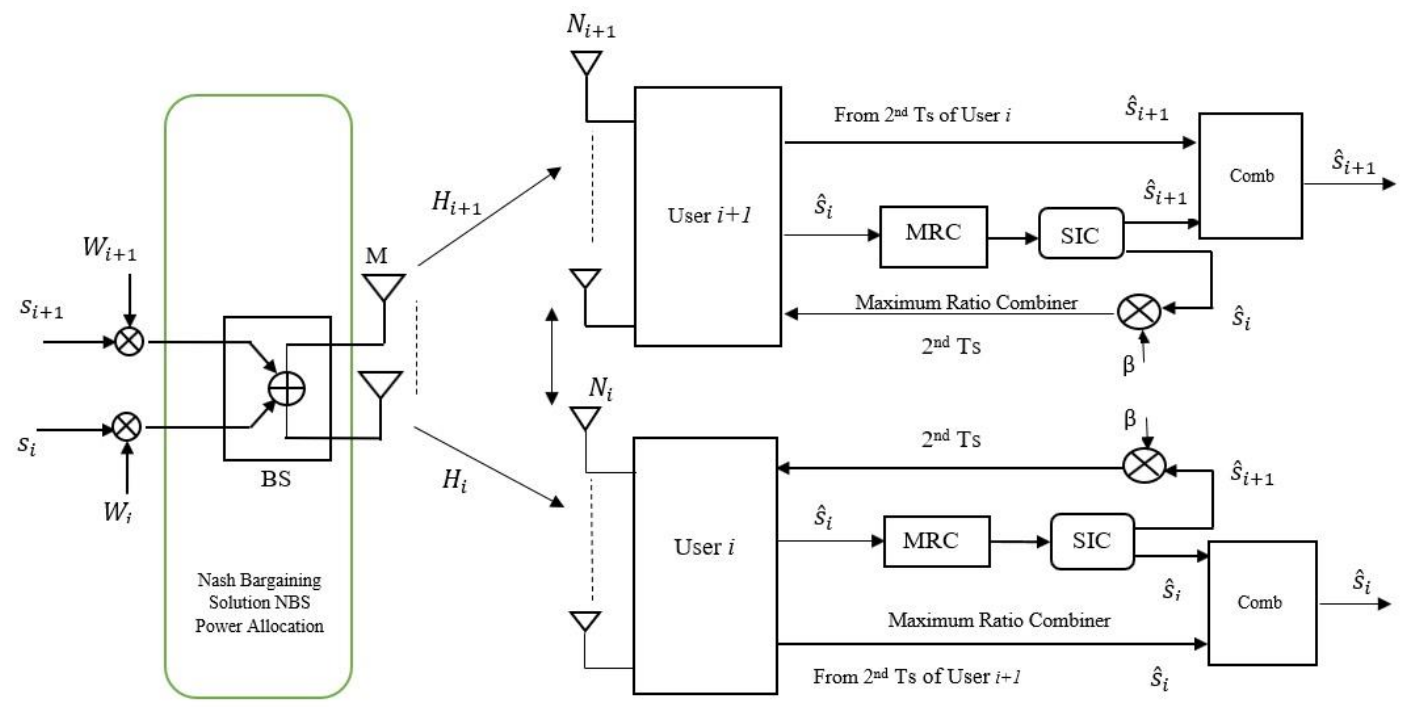

Fig. 3. MRC estimator for two-user full-duplex cooperative BF for MC of NOMA.

For signal detection at each user receiver and cooperation between them, the block diagram in Fig. 3 gives more explanation, where each user receives superposition signals that include first- and second-user signals.

\section{PROBLEM Formulation}

Finding an optimal power allocation strategy while considering the interference amongst the superposition of users' signals in NOMA is challenging. We aim to allocate $C$ subchannels and the transmitted power amongst $U$ users.

Therefore, we propose an optimal power allocation based on the NBS game scheme in full duplex cooperative beamforming based on SLR for MC of NOMA.

The mathematical model of the SLR beamforming approach is presented in this section. To understand the concept of SLR, we attempt to discuss the SLR analysis, which includes the maximization of SLR in Section A, then the SLR limit theorem will introduce in Section B, while the interference analysis will present in Section $\mathrm{C}$ as follow.

\section{A. SLR Analysis}

To understand the concept of SLR, we consider the single-user MRC shown previously in Fig. 3. Then, SINR is written as shown in Eq. (10). Using SINR in Eq. (10) for $i=$ $\{1 . \ldots U\}$ as an optimisation objective function for determining $\left\{w_{i}\right\}_{i=1}^{U}$ leads to a problem with $U$ coupled variables $\left\{w_{i}\right\}$.

$$
\operatorname{SINR}_{i}=\frac{E_{i}}{\sigma^{2}+\frac{\sum_{u=1, u \neq i}^{U}\left\|w_{i}^{*} \widetilde{H}_{i}^{*} \widetilde{H}_{i} w_{u}\right\|^{2} \alpha_{u}}{\left\|H_{i} w_{i}\right\|^{2}}}
$$


according to [27] for MRC,

$$
E_{i}=\alpha_{i}\left\|H_{i} w_{i}\right\|^{2}
$$

Now

$$
\mathbb{E}_{h}\left(E_{i}\right)=N \alpha_{i}\left\|H_{i} w_{i}\right\|^{2}
$$

where $E_{i}$ is desired signal power, while $\mathbb{E}_{h}\left(E_{i}\right)$ the expectation of the desired signals power, while $N$ is the number of transmitted antennas. Therefore, $\operatorname{SINR}_{i}$ will be,

$$
\operatorname{SINR}_{i}=\frac{N \alpha_{i}\left\|H_{i} w_{i}\right\|^{2}}{\sigma^{2}+\frac{\sum_{u=1, u \neq i}^{U}\left\|w_{i}^{*} \widetilde{H}_{i}^{*} \tilde{H}_{i} w_{u}\right\|^{2} \alpha_{u}}{\left\|H_{i} w_{i}\right\|^{2}}}
$$

The achievable rate of the $i^{\text {th }}$ user can be obtained as follows:

$$
R_{i}=\log _{2}\left(1+S I N R_{i}\right)
$$

Using this SINR expression for $i=\{1, \ldots, U\}$ as an optimization criterion for determining the $\left\{w_{i}\right\}$ would generally result in a problem with $U$ coupled variables $\left\{w_{i}\right\}$. In the sequel, in [20], they propose an alternative criterion to design the beamforming coefficients $\left\{w_{i}\right\}$, which leads to a full characterization of the optimal solutions in terms of generalized eigenvalue problems.

Let us reconsider Eq. (16). The power of the desired signal $H_{i} w_{i}$ is given by $\left\|H_{i} w_{i}\right\|^{2}$. At the same time, the power of the interference caused by this user $i$ on the signal received by user $u$ is given by $\left\|H_{i} w_{i}\right\|^{2}$. We define a quantity, called leakage for user $i$, as the total power leaked from this user to all other users:

$$
\sum_{u=1, u \neq i}^{U}\left\|H_{u} w_{i}\right\|^{2}
$$

Then SLR maximisation is performed to compute the maximum beamforming $\left(\mathrm{w}_{\mathrm{i}}^{\mathrm{o}}\right)$ for each user according to [20].

$$
w_{i}^{o}=\arg \max \frac{\left\|H_{i} w_{i}\right\|^{2}}{\sum_{u=1, u \neq i}^{U}\left\|H_{u} w_{i}\right\|^{2}}
$$

Subject to $\left\|w_{i}\right\|^{2}=w_{i}{ }^{H} w_{i}=P_{i} / E_{i}$

As shown in Eq. (16), and following our previous work [21], the power constraint proposed by [20] has been updated to $\left\|w_{i}\right\|^{2}=w_{i}{ }^{H} w_{i}=P_{i} / E_{i}$, the reason for this updating, is noted that the norm of $w_{i}$ is irrelevant to the final solutions, or in other words, the norm of $w_{i}$ can be forced to be any value to achieve the best value for $w_{i}$ under the power constraint. $P_{i} / E_{i}$ is the transmission power constraint at transmitter $i$, and it can be described as $E\left(\left\|w_{i} s_{i}\right\|^{2}\right) \leq$ 
$P_{i}$. The symbol $\mathrm{s}_{\mathrm{i}}$ satisfies the power constraint as $E_{i}=E\left(\left|s_{i}\right|^{2}\right)=1$.

By carefully examining Eq. (16). A key feature of the above criterion is that the design procedure for $w_{i}, i=\{1 \ldots . U\}$, involves $U$ is a decoupled optimisation problem compared with Eq. (10).

\section{B. Interference analysis}

It can be verified that the SLR expression in (16) can be rewritten as

$$
S L R=\frac{\left\|H_{i} w_{i}\right\|^{2}}{\left\|\widetilde{H}_{i} w_{i}\right\|^{2}}
$$

where

$$
\widetilde{H}_{i}=\left[\begin{array}{llllll}
H_{1}^{*} & \ldots & H_{i-1}^{*} & H_{i+1}^{*} & \ldots . . H_{U}^{*}
\end{array}\right]^{*}
$$

Equation (18), is the channel matrix which excludes $H_{i}$. where $H_{i} \in \mathbb{C}^{N \times M}$ represents the channel between the BS and user $u$ and $\widetilde{H}_{i}=\left[\begin{array}{llllll}H_{1}^{*} & \ldots & H_{i-1}^{*} & H_{i+1}^{*} & \ldots . . H_{U}^{*}\end{array}\right]^{*}$ denotes the corresponding leakage channel. The channel has been assumed to be a flat Rayleigh fading channel with a spatially uncorrelated. Moreover, $H_{i}$, and $\widetilde{H}_{i}$ are assumed a full rank matrix beside the probability is one. The transmitted symbol intended for $i^{t h}$ user $s_{i} \in \mathbb{C}^{M \times L}$ where $L$ $(\leq N)$ is the no. of data streams for $i^{t h}$ user which is assumed identical for entirely the users. The $s_{i}$ vector is satisfying the power constraint $E\left(s_{i} s_{i}{ }^{*}\right)=\mathbf{I}_{L} \cdot s_{i}$ is multiplied by a preceding matrix $w_{i}$. Then, for a given user $i$, the received signal vector:

$$
S L R=\frac{w_{i}^{*} H_{i}^{*} H_{i} w_{i}}{w_{i}^{*} \widetilde{H}_{i}^{*} \widetilde{H}_{i} w_{i}}
$$

The general solution of Eq. (19) that has been solved by [20] which obeys the RayleighRitz method [28]. Hence, we can solve Eq. (19) as:

$$
\frac{w_{i}^{*} H_{i}^{*} H_{i} w_{i}}{w_{i}^{*} \widetilde{H}_{i}^{*} \widetilde{H}_{i} w_{i}} \leq \lambda_{\max }\left(H_{i}^{*} H_{i}, \widetilde{H}_{i}^{*} \widetilde{H}_{i}\right)
$$

where $\lambda_{\max }$ is the largest generalised eigenvalue. Equality occurs when $w_{i}$ is proportional to a generalised eigenvector that corresponds to the largest generalised eigenvalue; compactly written as:

$$
w_{i}^{o} \propto \text { maxgen. eigenvector }\left(H_{i}^{*} H_{i}, \widetilde{H}_{i}^{*} \widetilde{H}_{i}\right)
$$

where $w_{i}^{o}$ is the maximal SLR. In the next section, we refer to the maximal SLR $\left(w_{i}^{o}\right)$ for each user by using parameter $\gamma_{i} . \alpha_{i}$ is the power allocation for the $i^{\text {th }}$ user.

\section{SLR limit theorem}

In (16), the SLR problem statement constrain [20] will allocate a fixed transmit power for each user, design $w_{i}, i=\{1, \ldots, U\}$, such that the signal-to-leakage ratio (SLR) is 
maximized for every user. In this paper we update the SLR problem statement constrain to new form as shown:

$$
\left\|w_{i}\right\|^{2}=w_{i}{ }^{H} w_{i} \leq P_{i} / E_{i}
$$

The reason for this updating is the drawback of the constraint in the problem statement (16) when each user has multiple data streams, the effective channel gain for each stream can be severely unbalanced. If power control or adaptive modulation and coding cannot be applied, the overall error performance of each user will suffer significant loss [29].

It is noted that the norm of $w_{i}$ is irrelevant to the final solutions, or in other words, the norm of $w_{i}$ can be forced to be any value to achieve the best value for $w_{i}$ under the power constraint shows in the problem statement (16).

\section{BARGAINING SOLUTION}

\section{A. Power Allocation Based on NBS Game Theory}

The power allocation based on the NBS game theory can be decomposed into three problems. The first subproblem is the optimization problem, where the maximizing of the utility function (which is a function of achievable rate) is achieved. The second problem is the existence of NBS, where the Hessian matrix will be used to approve the concave suffices of the utility function. The third problem is the power allocation scheme using NBS, where the Lagrangian method will be used to solve the constrained optimization problems. The power allocation based on the NBS game theory block diagram is shown in Fig. 4. 


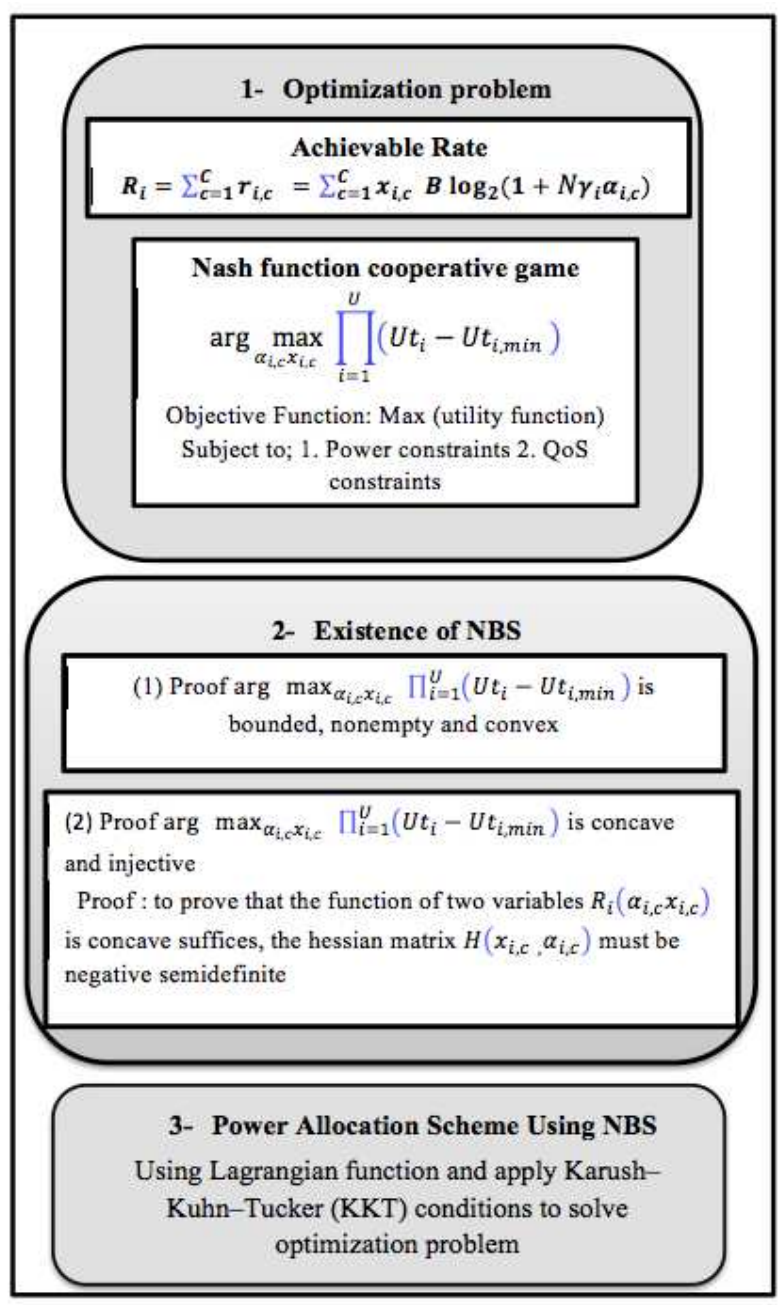

Fig. 4. Problem formulation of the power allocation based on NBS game

\section{B Optimization problem}

With the preliminaries of max-SLR $\left(\gamma_{i}\right)$ in the previous section, a new formula for optimization problem with the help of NBS has been formulated. As aforementioned, both users seek help from another user to enhance the performance. The utility of the user depends on two factors. One is the channel conditions of the cooperative link between cooperating users. The other one is how much leakage power would the selected user split for the relay. Intuitively, to maximize far user performance, BS users would like to invite the user who owns good channel quality to join the cooperation and expect him to support as much power as possible to support the information relaying. While the nearest user who is involved in the game, gains more power from leakage power from far users through cooperation, at the cost 
of sacrificing power to relay the signal of the far user and interference experienced from far user. Therefore, the utility $U t_{i}$ for $i^{\text {th }}$ user is defined as [30]:

$$
U t_{i}=R_{i}-\gamma_{u} \alpha_{u, c}
$$

where $\gamma_{u}$ is the max-SLR and $\alpha_{u, c}$ is the power allocated for $u^{t h}$ user on subchannel $c$, where $\alpha_{u, c}+\alpha_{i, c}=1$, while $R_{u}$ is the achievable rate for the $u^{t h}$ user. The achievable rate $R_{i}$ for the $i^{\text {th }}$ user, which is the sum of the rates in each subchannel, as follows [31]:

$$
R_{i}=\sum_{c=1}^{C} r_{i, c}=\sum_{c=1}^{C} x_{i, c} B \log _{2}\left(1+N \gamma_{i} \alpha_{i, c}\right)
$$

By plugging equation (23) in equation (24) and replace $\alpha_{u, c}=1-\alpha_{i, c}$ the utility for $i^{\text {th }}$ user will be:

$$
U t_{i}=\sum_{c=1}^{C} x_{i, c} B \log _{2}\left(1+N \gamma_{i} \alpha_{i, c}\right)-\gamma_{u}\left(1-\alpha_{i, c}\right)
$$

where $\gamma_{i}$ is the max-SLR and $\alpha_{i, c}$ is the power allocation of the $i^{\text {th }}$ user on subchannel $c$. while $x_{i, c}$ is subchannel assignment coefficient.

Resource allocation (optimal power and channel allocation) is our target. The resource allocation problem is to allocate $C$ subchannels and the transmitted power amongst $U$ users, so that the maximum throughput is achieved.

In NOMA, the nearest user (best user channel) is assigned with less power, while the far user (worst user channel) is assigned with more transmission power. More explicitly, the priority for subchannel assigning follows the channel condition $H_{i-1} \leq H_{i}$ to achieve a certain fairness objective. Assume the subchannel assignment coefficient is $\left(x_{i, c}\right)$ [32].

$$
x_{i, c}=\left\{\begin{array}{lc}
1 & \text { if user } i \text { are multiplexed on subcarrier } c \text { with } H_{1} \leq H_{i+1} \\
0 & \text { otherwise }
\end{array}\right.
$$

$x_{i, c}$ is given as optimal joint power and subcarrier allocation for full-duplex multicarrier nonorthogonal multiple access systems. 
$H_{i+1}$ represent the nearest user channel condition (best user channel).

$H_{i}$ represent the farther user channel condition (worst user channel)

Assume the number of subcarriers is five, because NOMA implementation considers that the decoding complexity and signaling overhead increase with the no. of subcarrier.

Unlike the OFDMA, which assumed each subcarrier is assigned for one user, NOMA assumed that all available subcarriers are used by all users at the same time and frequency by exploiting the distinguish in power level. More explicitly, NOMA enables each user to have access to all the subcarrier channels, and hence the bandwidth resources allocated to the users with poor channel conditions can still be accessed by the users with strong channel conditions, which significantly improves the spectral efficiency, but the problem is who this BS should be assigned the available power between users, so the overall performance is optimized. For this reason, cooperative NOMA-based NBS is suggested to offer negotiation between users via the BS.

The bargaining problem is contained from the utility function $U t_{i}$ of user $i^{\text {th }}$ as mentioned in equation (25), which is a function of the set containing all the feasible rates $S$, and $U t_{i, \min }$ is the minimum rate $R_{i, \min }$ which is decided by the disagreement point. The NB solution can be derived by solving the following bargaining optimization problem:

$$
\begin{gathered}
\text { NBS: } F=\arg \max _{\alpha_{i, c} x_{i, c}} \prod_{i=1}^{U}\left(U t_{i}-U t_{i, \min }\right) \\
\text { subject to } C_{1}: \sum_{i=1}^{U} \sum_{c=1}^{C} \alpha_{i, c} \leq P_{\max }, C_{2}: R_{i} \geq R_{i, \text { min }}, \\
C_{3}: \alpha_{i, c} \geq 0, C_{4}: \sum_{i=1}^{U} x_{i, c}=1, C_{5}: x_{i, c} \in\{0,1\}
\end{gathered}
$$

The objective function in (27) is the Nash function. The first constraint is the requirement to guarantee the available power for all users is bound by the total power constraints of the base station $P_{\max }$. The second constraint is to ensure the minimum rate 
requirement, while the fourth constraint states the subchannel will be assigned for each user if and only if $H_{i-1} \leq H_{i}$, therefore NB solution exists if and only if the far user are assigned with more power then all users can benefit from the NOMA-based cooperation. This optimisation problem is difficult to solve because it deals with both continuous and binary variables, so an approach is suggested to relax the condition in $C_{5}$ by permitting $x_{i}$ take values between $[0,1]$.

\section{Existence of NBS}

The main challenge of using NBS with NOMA is that the interference amongst the superposition of users' signals in the NOMA environment causes a non-convex utility space (rate region) [19]. Orthogonal signaling converts the non-convex utility space to a convex one, limiting the use of NBS with NOMA. Hence, the Game Theory is used to allocate power to each user in the NOMA environment with SLR precoding.

Theorem: The Nash bargaining (NB) exists if function (27) satisfies two main conditions:

1- The utility set $S$ defined in function (27) is a closed and bounded convex subset.

2- The utility function $R_{i}$ is a concave down function and injective.

Proof: it is straightforward to prove that the above conditions are satisfied with the following states

1- The set is convex because the constraints of the optimisation problem are linear. On another side, the maximal value of SLR improves the power level of the desired user while reducing the interference to other users from the desired user, thus leading to orthogonality and converts the non-convex utility space to a convex one. Hence the first condition is easily satisfied.

2- In order to show that the second state is also satisfied, then the defined of function (27) should be proved to be concave. 
To show that the second condition is true, the following set of equations should be solved $\frac{\partial^{2} r_{i, c}}{\partial \alpha_{i, c}^{2}}<0$. In the following:

$$
\begin{gathered}
\text { Let } R_{i}=\sum_{c=1}^{C} r_{i, c} \\
H\left(x_{i, c}, \alpha_{i, c}\right)=\left(\begin{array}{cc}
\frac{\partial^{2} r_{i, c}}{\partial \alpha_{i, c}^{2}} & \frac{\partial^{2} r_{i, c}}{\partial \alpha_{i, c} \partial x_{i, c}} \\
\frac{\partial^{2} r_{i, c}}{\partial \alpha_{i, c} \partial x_{i, c}} & \frac{\partial^{2} r_{i, c}}{\partial x_{i, c}^{2}}
\end{array}\right) \\
\frac{\partial r_{i, c}}{\partial \alpha_{i, c}}=\frac{N x_{i, c} B \gamma_{i}}{\ln 2\left(1+N \gamma_{i} \alpha_{i, c}\right)} \\
\frac{\partial^{2} r_{i, c}}{\partial \alpha_{i, c}^{2}}=\frac{-x_{i, c} B \gamma_{i}^{2} N^{2}}{\ln 2\left(1+N \gamma_{i} \alpha_{i, c}\right)^{2}} \\
H\left(x_{i, c}, \alpha_{i, c}\right)=\left(\begin{array}{ll}
\frac{-x_{i, c} B \gamma_{i}^{2} N^{2}}{\ln 2\left(1+N \gamma \alpha_{i, n}\right)^{2}} & 0 \\
0 & 0
\end{array}\right)
\end{gathered}
$$

From solving the Hessian matrix in above equation (31) the first element $\left(\frac{-x_{i, c} B \gamma_{i}{ }^{2} N^{2}}{\ln 2\left(1+N \gamma_{i} \alpha_{i, n}\right)^{2}}\right)$ is $<0$, so that $H\left(x_{i, c}, \alpha_{i, c}\right)$ is negative semidefinite. Therefore, utility function is concave.

\section{Power Allocation Scheme Using NBS}

One of the efficient methods that can be applied to solve the constrained optimization problems, which is shown in equation (27) is the Lagrangian method, where $\left(\lambda, v_{i, c}, \mu_{c}\right)$ is the multiplier, vector. Furthermore, the Karush-Kuhn-Tucker (KKT) conditions are applied in order to find the optimal solution of equation (27) [33], and by replacing $\alpha_{u, c}=1-\alpha_{i, c}$ to find the optimal power allocated for user $\alpha_{i, c}$ :

$$
\begin{aligned}
& L\left(\alpha_{i, c}, x_{i, c}\right)=\left(\sum_{c=1}^{C} x_{1, c} B \log _{2}\left(1+N \alpha_{1, c} \gamma_{1}\right)-\gamma_{u}\left(1-\alpha_{1, c}\right)-\right. \\
& \left.R_{1, \min }\right) \ldots \\
& \ldots\left(\sum_{c=1}^{C} x_{i, c} B \log _{2}\left(1+N \alpha_{i, c} \gamma_{i}\right)-\gamma_{u}\left(1-\alpha_{i, c}\right)-R_{1, \min }\right)
\end{aligned}
$$




$$
\begin{gathered}
-\lambda \sum_{i=1}^{U}\left(\sum_{c=1}^{C} \alpha_{i, c}-P_{\max }\right)-\sum_{i=1}^{U} \sum_{c=1}^{C} v_{i, c} \alpha_{i, c}- \\
-\sum_{c=1}^{C} \mu_{c}\left(\sum_{i=1}^{U} x_{i, c}-1\right)
\end{gathered}
$$

KKT condition are:

$$
\begin{aligned}
& \frac{\partial L\left(\alpha_{i, c}, x_{i, c}\right)}{\partial \alpha_{i, c}}=0 \\
& \frac{\partial L\left(\alpha_{i, c}, x_{i, c}\right)}{\partial x_{i, c}}=0 \\
& \lambda \sum_{i=1}^{U}\left(\sum_{c=1}^{c} \alpha_{i, c}-P_{\max }\right)=0 \\
& \sum_{c=1}^{C} \mu_{c}\left(\sum_{i=1}^{U} x_{i, c}-1\right)=0 \\
& \lambda_{i,} v_{i, c} \mu_{c} \geq 0 \\
& \alpha_{i, c} v_{i, c}=0
\end{aligned}
$$

apply $\frac{\partial L\left(\alpha_{i, c}, x_{i, c}\right)}{\partial \alpha_{i, c}}=0$ from equation (33):

$$
\begin{gathered}
\prod_{j=1, j \neq i}^{U}\left(R_{j}-R_{j, m i n}\right) x_{i, c} B \frac{N \gamma_{i}}{1+N \gamma_{i} \alpha_{i, c}}-\lambda+\gamma_{u}=0 \\
\frac{\prod_{j=1, j \neq i}^{U}\left(R_{j}-R_{j, m i n}\right) x_{i, c} B \gamma_{i} N}{1+N \gamma_{i} \alpha_{i, c}}=\lambda-\gamma_{u} \\
\frac{1+N \gamma_{i} \alpha_{i, c}}{\prod_{j=1, j \neq i}^{U}\left(R_{j}-R_{j, \min }\right) x_{i, c} B \gamma_{i} N}=\frac{1}{\lambda-\gamma_{u}} \\
1+N \gamma_{i} \alpha_{i, c}=\frac{\prod_{j=1, j \neq i}^{U}\left(R_{j}-R_{j, \min }\right) x_{i, c} B \gamma_{i} N}{\lambda-\gamma_{u}} \\
N \gamma_{i} \alpha_{i, c}=\frac{\prod_{j=1, j \neq i}^{K}\left(R_{j}-R_{j, \min }\right) x_{i, c} B \gamma_{i} N}{\lambda-\gamma_{u}}-1 \\
\alpha_{i, c}=\frac{\frac{\prod_{j=1, j \neq i}^{U}\left(R_{j}-R_{j, \min }\right) x_{i, c} B \gamma_{i} N}{\lambda-\gamma_{u}}}{N \gamma_{i}}-\frac{1}{N \gamma_{i}}
\end{gathered}
$$

Therefore, the solution of equation (27) produces the following power allocation formula $\alpha_{i, c}$ : 


$$
\alpha_{i, c}=\frac{\prod_{j=1, j \neq i}^{U}\left(R_{j}-R_{j, \min }\right) B}{\lambda-\gamma_{u}}-\frac{1}{N \gamma_{i}}
$$

Assume that subchannel $c$ is assigned to user $i$ according to constraint $C_{4}, x_{i, c}=1$.

As shown above, equation (45) has the familiar shape of a water filling equation with slight changes in the water level. Therefore, more power is allocated to the subchannels with smaller gains.

After the subchannels are allocated ( $x_{i, c}$ is known), from equation (45) and equation (35), then by apply $\frac{\partial L\left(\alpha_{i, c}, x_{i, c}\right)}{\partial \lambda}=0$.

$$
\frac{\partial L\left(\alpha_{i, c} x_{i, c}\right)}{\partial \lambda}=p_{\max }-\sum_{i=1}^{U} \alpha_{i, c}
$$

and from substitute equation (45) in equation (46):

$$
p_{\max }-\left(\sum_{i=1}^{U} \frac{\mathcal{W} B}{\lambda-\gamma_{u}}-\frac{1}{N \gamma_{i}}\right)=0
$$

where

$$
\begin{gathered}
\mathcal{W}=\prod_{j=1, j \neq i}^{U}\left(\left(R_{j}-R_{j, \text { min }}\right)\right. \\
p_{\text {max }}+\sum_{i=1}^{U} \frac{1}{N \gamma_{i}}=\frac{B \mathcal{W}}{\lambda-\gamma_{u}}
\end{gathered}
$$

The total bandwidth $B$ is divided into $C$ subchannels, each with bandwidth $\frac{B}{C}$. Then, 


$$
\alpha_{i, c}=\frac{p_{\max }+\sum_{i=1}^{U} \frac{1}{N \gamma_{i}}}{C}-\frac{1}{N \gamma_{i}}
$$

where $C$ is the number of subcarriers assigned for each user.

The results of equation (49) are similar to those of a water filling equation. Searching over $U \times C$, the subchannel to noise ratio matrix reduces the complexity from $O\left(U^{2}\right)$ to $U \times$ C.

$$
\begin{aligned}
& \text { By applying equation } 34, \frac{\partial L\left(\alpha_{1, c} x_{1, c}\right)}{\partial x_{1, c}}=0 \\
& \left(\log _{2}\left(1+N \gamma_{1} \alpha_{1, c}\right)-\gamma_{2}\left(1-\alpha_{1, c}\right)\right)\left(R_{2}-R_{2, \min }\right)=0 \\
& \frac{\partial L\left(\alpha_{2, c} x_{2, c}\right)}{\partial x_{2, c}}=0 \\
& \left(\log _{2}\left(1+N \gamma_{2} \alpha_{2, c}\right)-\gamma_{1}\left(1-\alpha_{2, c}\right)\right)\left(R_{1}-R_{1, \min }\right)=0 \\
& \frac{\partial L\left(\alpha_{i, c}, x_{i, c}\right)}{\partial x_{i, c}}=\frac{\partial L\left(\alpha_{1, c} x_{1, c}\right)}{\partial x_{1, c}}=\frac{\partial L\left(\alpha_{2, c} x_{2, c}\right)}{\partial x_{2, c}} \\
& \left(\log _{2}\left(1+N \gamma_{1} \alpha_{1, c}\right)-\gamma_{2}\left(1-\alpha_{1, c}\right)\right)\left(R_{2}-R_{2, \min }\right) \\
& =\left(\log _{2}\left(1+N \gamma_{2} \alpha_{2, c}\right)-\gamma_{1}\left(1-\alpha_{2, c}\right)\left(R_{1}-R_{1, \min }\right)\right. \\
& \frac{\left(\log _{2}\left(1+N \gamma_{1} \alpha_{1, c}\right)-\gamma_{2}\left(1-\alpha_{1, c}\right)\right)}{\left(R_{1}-R_{1, \min }\right)} \\
& =\frac{\left(\log _{2}\left(1+N \gamma_{2} \alpha_{2, c}\right)-\gamma_{1}\left(1-\alpha_{2, c}\right)\right)}{\left(R_{2}-R_{2, \min }\right)} \\
& \left(R_{1}-R_{1, \min }\right) \\
& \frac{\left(\log _{2}\left(1+N \gamma_{i} \alpha_{i, c}\right)-\gamma_{u}\left(1-\alpha_{i, c}\right)\right)}{\left.\left(1+N \gamma_{1} \alpha_{1, c}\right)-\gamma_{2}\left(1-\alpha_{1, c}\right)\right)} \\
& \left(R_{1}\right)
\end{aligned}
$$

The finding of $\alpha_{i, c}$, and $x_{i, c}$ from the solution of equation (49) and equation (56) provides an optimization problem solution for NP-hard problem, comparing with [33], the final solution of our work provides the formula for the optimum solution for NOMA. In contrast, the optimization problem of [33] provide solution of OFDM, which consider OMA 
system. More explicitly in our work the optimal power value $\alpha_{i, c}$ will be used as a power level domain in the cooperative NOMA system by employing SIC estimator at the receiver, while [33] did not use the optimal power in any estimation technique. Furthermore, in our work, the SLR beamforming technique has been used to enhance system performance by employing the value of $\gamma_{i}$ (which was solved in equation 21) in the rate equation. On the other hand, our system considers a cooperative environment, while [33] considered a noncooperative environment.

Eq. (56) shows that the ratio of the rate by assigning one subchannel to the total rate should be the same for all users. This idea emphasizes the fairness of the optimal solution and gives us a metric of allocating subchannels.

\section{Simulation Setting ANd Results}

\section{A. BER Performance}

The proposed NBS power allocation full-duplex cooperative BF for MC of NOMA (proposed scheme) introduced in Section II is simulated using Matlab codes. The simulation considers two time slots. In the first time slot, the BS broadcasts a superposition of individual signals to multiple users' receivers over a Rayleigh fading channel with zero mean. In the second time slot, both users' channels cooperate with each other over a Rician fading channel with $m$ mean (inter-user channels), unit variance and i.i.d complex Gaussian random variables. The summary of simulation parameters is shown in Table I.

TABLE I SIMULATION PARAMETERS

\begin{tabular}{lc}
\hline \hline Parameters & Definition \\
\hline Modulation mode & QPSK \\
No. of input data & 100000 \\
BER comparison point & $10^{-5}$ \\
Downlink channel & Rayleigh \\
Inter-user channels & Rician \\
SNR of inter-user channel & 20 \\
Number of users $(U)$ & 2 \\
Number of antennas for BS $(M)$ & $4,6,8,16$ \\
Number of antennas for each user $(N)$ & $4,6,8.16$ \\
Number of subcarriers [3][11] & 5 \\
Rician channel factor $(K d B)$ & 30 \\
Beta $(\beta)$ & 0.1 \\
\hline \hline
\end{tabular}

According to [23] the NOMA's BER performance of all the systems described are evaluated at a BER of $10^{-5}$. An acceptable BER performance for voice communication is $10^{-3}$ while, that for data transmission is $10^{-5}$ [34]. All the simulated results carried out at $\beta$ $=0.1$, which offer better system performance, where $B$ is a constant to meet the total 
transmitted power constraint [27]. Assuming the nearest user is encouraged to cooperate with the best line of sight (LOS), the Rician channel factor is taken at $K=30 \mathrm{~dB}$ in all simulated results [35]. While the inter-user channel is considered at $S N R=20 d B$, which show an enhancement in system performance comparing with lowest than $20 \mathrm{~dB}$ cases, since in second time slot interference due to concurrently communicating users will increase, which mean inter-user interference increase IUI, resulting in poor system performance signal [36][37].

To evaluate the performance of the proposed scheme, we have implemented a previous multiple-input and multiple-output MIMO-NOMA [23] scheme with $M=N=4$ antenna, also the BER performance of the proposed scheme is compared with that of the non-cooperative scheme, as shown in Fig. 5.

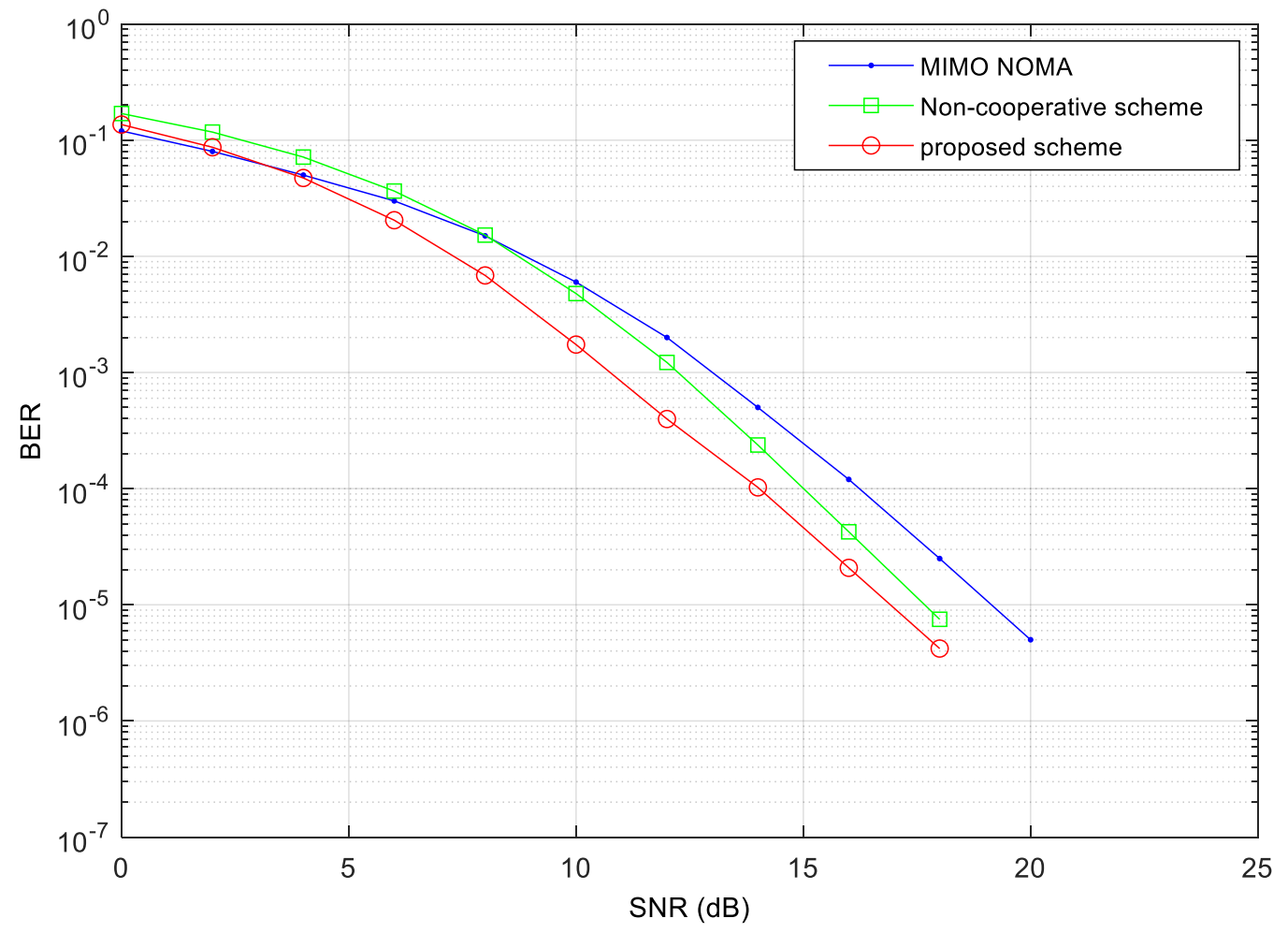

Fig. 5. Comparison of the BER performance of the proposed scheme, non-cooperative scheme and MIMONOMA [23] with $K d B=30$ at inter-user channel $S N R=20$, for $M=N=4$ antenna and $U=2$ users.

Fig. 5 shows the comparison of the BER performance of the proposed scheme, Noncooperative scheme and MIMO-NOMA [23]. The result demonstrates that the performance of the proposed scheme is better than that of the non-cooperative scheme since our proposed scheme turns the interference signals (second user signal ) into valuable signals after detecting and separating these signals by SIC. Specifically, to achieve a BER of about $10^{-5}$, the required SNR for proposed scheme is about $2 d B$ less than that for non-cooperative scheme. Compared with the other work, the proposed scheme is better than MIMO-NOMA [23]. Specifically, to achieve a BER of about $10^{-5}$, the required SNR for the proposed scheme is about $3 d B$ less than that for MIMO-NOMA [23]. 
To show the effect of inter-user channel on system performance Fig.6, presented the performance of the proposed under different values of SNR inter-user channel $(5,10,15$ and $20 \mathrm{~dB}$ ). The performance of the system will be enhanced when inter-channel SNR increases, as the increasing in SNR of inter-user channel intended more average power of the desired signal are allocated to each cooperative user and the effect of Inter-user interference IUI will decrease.

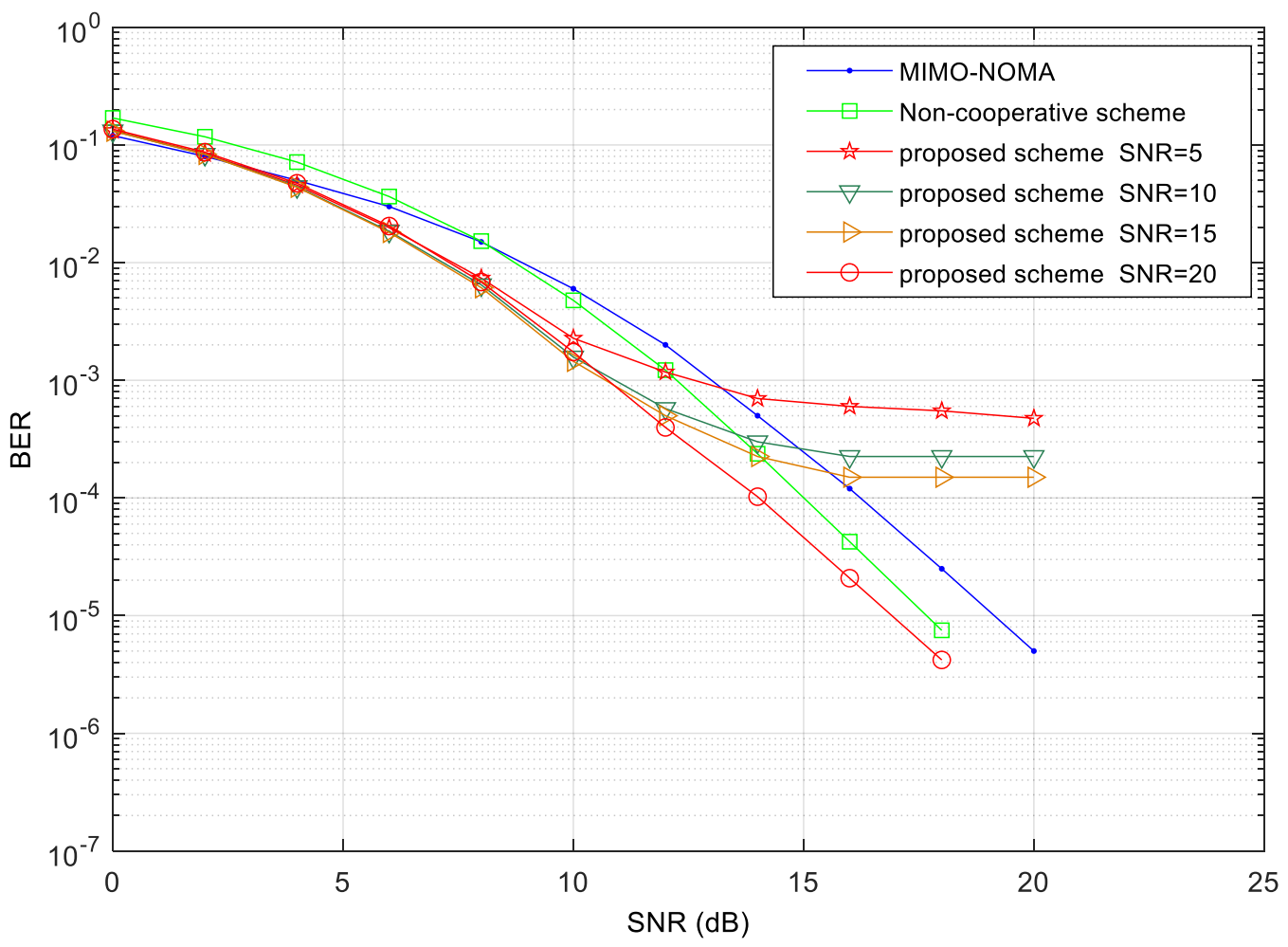

Fig. 6. Comparison of the BER performance of the proposed scheme under different values of inter-user channel $\left(S N R_{\text {int }}=5,10,15\right.$ and $\left.20 \mathrm{~dB}\right)$ for $M=N=4$ antenna and $U=2$ users. 


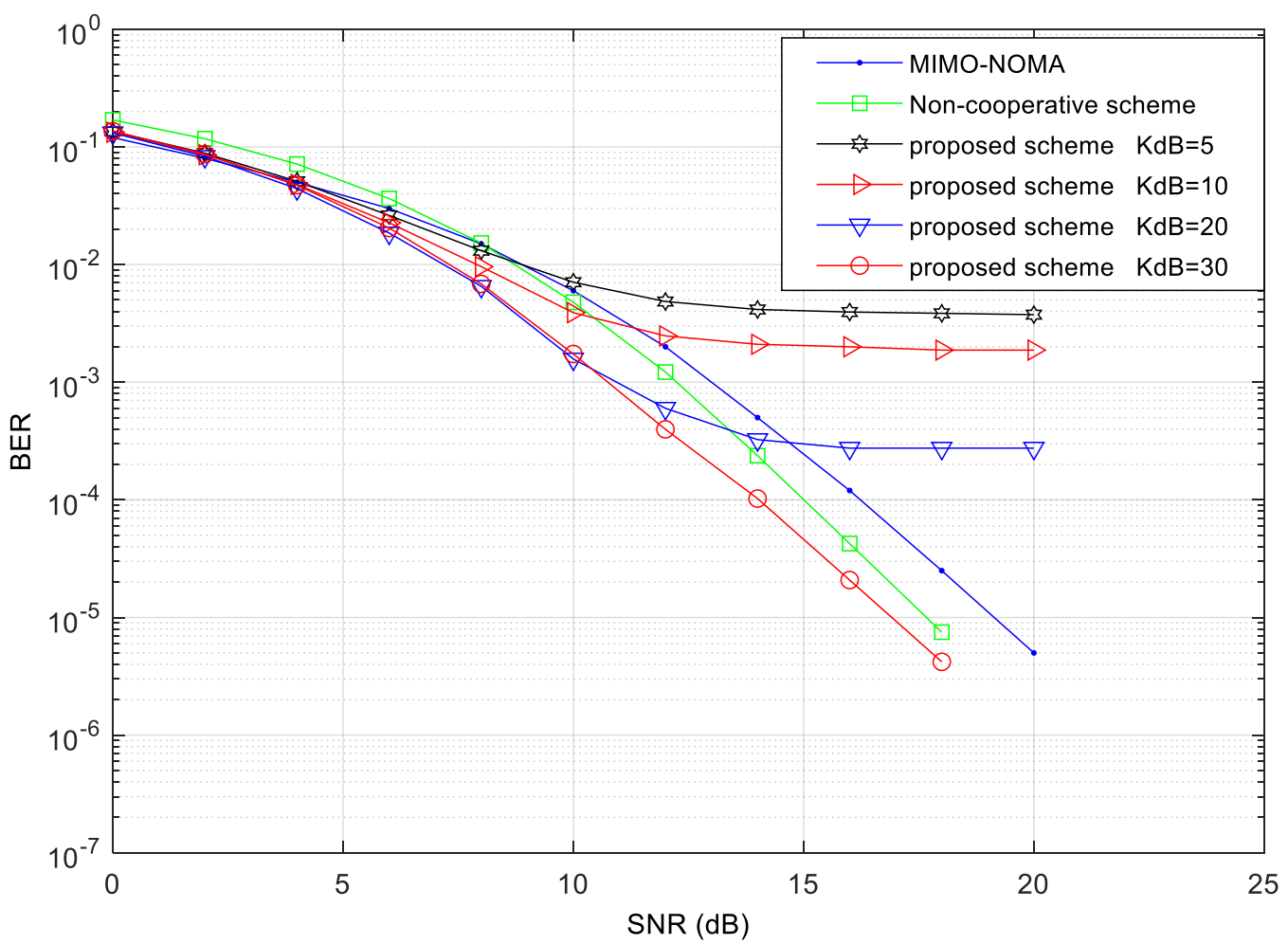

Fig. 7. Comparison of the BER performance of proposed scheme under different values of $K d B=5,15$ and 20 for $M=N=4$ antenna and $U=2$ users.

Fig. 7 shows the system performance when the inter-user channel uses a LOS environment (over a correlated realistic Rician fading channel). The performance of the proposed scheme is enhanced as $K d B$ increases. Specifically, in case $K d B=30$, to achieve a BER of about $10^{-3}$, the required SNR for the proposed scheme is about $3 d B$ less than that for the noncooperative scheme. In other words, when the inter-user channel LOS is reduced, the total proposed system performance is also reduced. since the user will not be LOS, which means the effect of IUI will increase. 


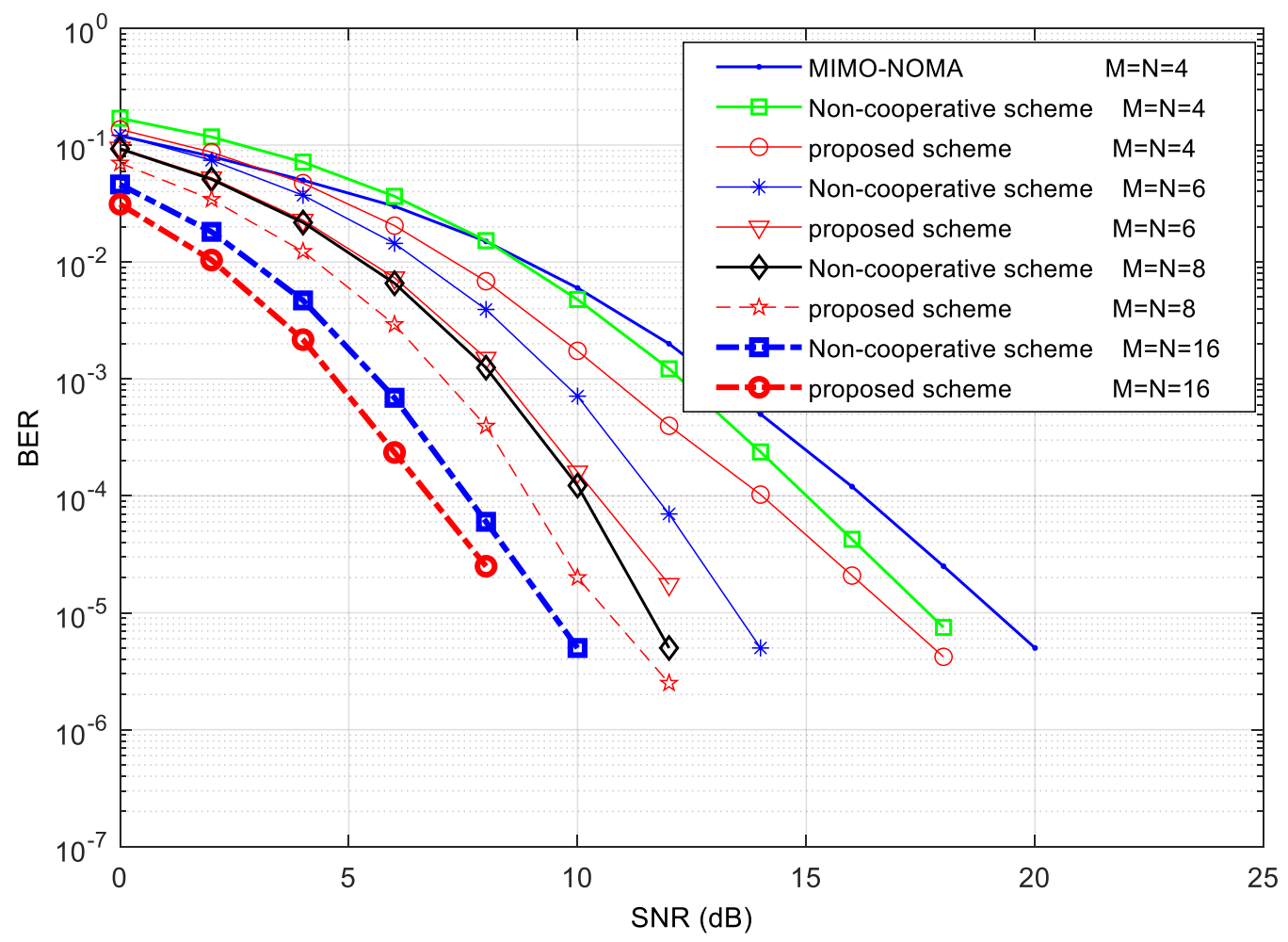

Fig. 8. Comparison of the BER performance of the proposed scheme and non-cooperative scheme using 4, 6, 8 and 16 antennas in both the BS and users with $K d B=30$ at inter-user channel $\mathrm{SNR}=20$ for and $U=2$ users.

Fig. 8 shows a comparison of the BER performance of the proposed scheme and the noncooperative scheme employed multi-antennas in both the BS and users, where the BS antennas $M=4,6,8$ and 16 and users' antennas $N=4,6,8$ and 16. The result shows that the system exhibits a significant improvement for $M=16, N=16$ in both non-cooperative and the proposed scheme, because of our proposed scheme is designed to support a high number of antenna.

\section{B. Fairness Performance}

In this part, we evaluate the performance of the proposed scheme in fairness. To evaluate the performance of the proposed scheme, we have simulated the previous work [9], which has been improved "fractional transmit power control" FTPC and channel allocation model for both non-orthogonal (NOMA) and orthogonal frequency multiple access schemes (NOMAFTPC), (OFDMA-FTPC) respectively.

In power allocation based on FTPC [9], the user with lower channel condition assigned with more power to grantee the fairness between users, while the greed-based user technique has been employed to assign each subcarrier $c$ for the users $U$. Moreover, the performance of the proposed scheme has been evaluated with the model of power and channel allocation in 
NOMA [11], that has been combined the Lagrangian duality and dynamic programming (LDDP) to find near-optimal N- LDDP solutions.

Based on the LTE Standard, the bandwidth of the OFDMA-FTPC has been fixed to $4.5 \mathrm{MHz}$, which content 25 subchannels, each subchannel has $180 \mathrm{kHz}$ bandwidth. While in NOMA, 5 subcarriers are considered and used bandwidth of $900 \mathrm{kHz}$ for each in NOMA-FTPC, where decoding complexity and signalling overhead will be increased with the no. of subcarriers [38]. In our proposed scheme, Following the NOMA setup in [9], we assumed 5 subcarriers are assigned for each user.

In communications networks, the measure of fairness for user throughput follows Jain's fairness index [39]. The slandered form for calculating the Jain's fairness index is $\frac{\left(\sum_{u=1}^{U} \bar{R}_{u}\right)^{2}}{U \sum_{u=1}^{U}{\overline{R_{u}}}^{2}}$, the $\overline{R_{1}}, \ldots, \overline{R_{u}}$, denote the average users' rates. Jain's fairness index is examined between the value between $\frac{1}{U}$ and 1.0. The peak value means the system has a fairer throughput distribution. The maximum value, which is equal to 1.0 is achieved when all users achieve the same throughput. Note that the usage of this index, by himself, does not avoid the user from being served with low throughput (or zero throughputs), which, maybe lead down the value of the index. The fairness index in respect of $U$ is shown in Fig. 9.

From Fig. 9, we observe that, first, the proposed scheme achieves the best performance, the fairness index increases in $U$. Since a larger $U$ provides more flexibility in resource allocation among the users.

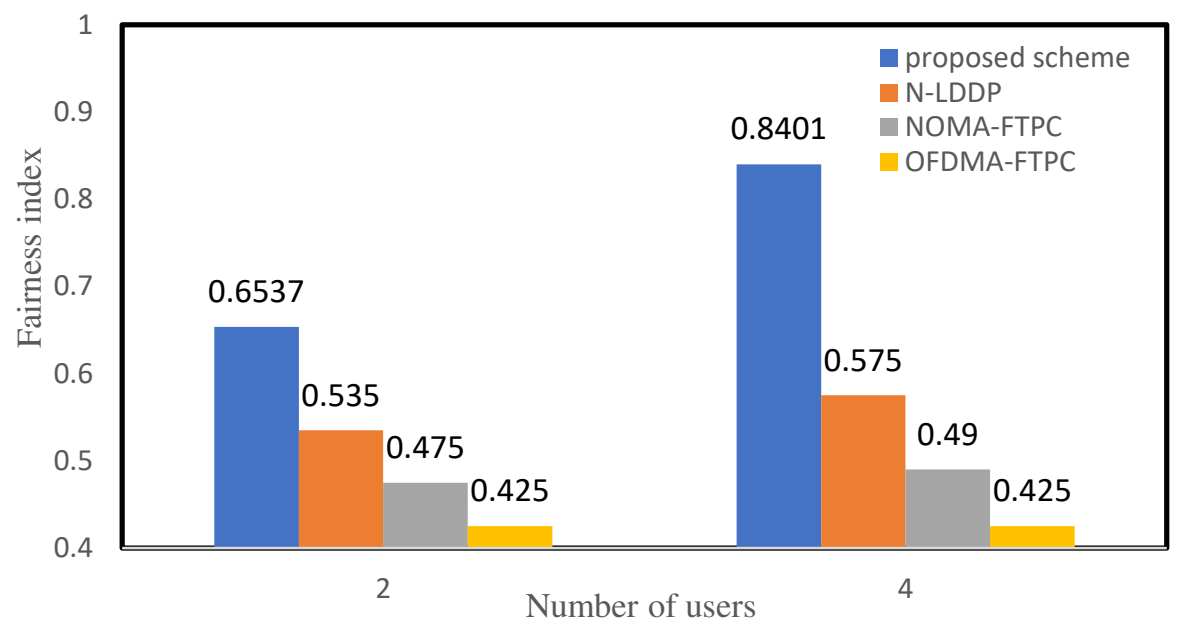

Figure 9. Fairness comparison in respect of the number of users.

From the result, OFDMA-FTPC gives the lowest fairness index. The reason is that the FTPC channel and power allocation scheme is sub-optimal. This also explains the improvement enabled by the proposed power optimization algorithm in comparison to NOMAFTPC and NLDDP. 


\section{CONCLUSIONS}

A fair scheme of power and channel allocation based on NBS for full-duplex cooperative $\mathrm{BF}$ for MC of NOMA. is proposed. The proposed scheme assigns optimal power and channel allocation according to channel conditions while keeping a fair rate amongst cooperative users. Meanwhile, NBS offers a fair and optimum approach to maximise the total rate of the CNOMA system. The SLR precoding technique is used to design a beamforming vector for achieving the power domain between the superposition users' signals. NBS offers a fair and optimum approach to maximise the total rate of the NOMA system. Simulation results show that the NBS power allocation in the proposed scheme improves the BER performance and fairness gain, compared with the non-cooperative scheme and MIMO-NOMA.

\section{DECLARATIONS}

The authors declare there is no conflict of interest on the publication.

\section{REFERENCES}

[1] Z. Ding, Z. Yang, P. Fan, and H. V. Poor, "On the performance of non-orthogonal multiple access in $5 \mathrm{G}$ systems with randomly deployed users," IEEE Signal Process. Lett., vol. 21, no. 12, pp. 1501-1505, Dec. 2014.

[2] S. M. R. Islam, N. Avazov, O. A. Dobre, and K. s. Kwak, "Power-domain nonorthogonal multiple access (NOMA) in 5G systems: Potentials and challenges," IEEE Commun. Surveys Tutorials, vol. 19, no. 2, pp. 721-742, Secondquarter 2017.

[3] X. Su, H. Yu, W. Kim, C. Choi and D. Choi, "Interference cancellation for nonorthogonal multiple access used in future wireless mobile networks", EURASIP J. Wireless Commun. Netw., 2016.

[4] O. Abbasi and A. Ebrahimi, "Cooperative NOMA with full-duplex amplify-andforward relaying," Trans. Emerg. Telecommun. Technol., vol. 29, no. 7, p. e3421, Jul. 2018.

[5] N.Dahi, N. Hamdi, "Outage Performance of Generalized Cooperative NOMA Systems with SWIPT in Nakagami-m Fading," Journal of Communications Software and Systems, vol. 14, no. 4, december 2018.

[6] M. Liaqat, K. A. Noordin, T. A. Latef, and K. Dimyati, "Power-domain non orthogonal multiple access (PD-NOMA) in cooperative networks: an overview," Wireless Networks, pp. 1-23, 2018.

[7] R. Jiao, L. Dai, J. Zhang, R. MacKenzie, and M. Hao, "On the performance of NOMAbased cooperative relaying systems over Rician fading channels," IEEE Trans. Veh. Technol., vol. 66, no. 12, pp. 11409-11413, Dec. 2017

[8] Y. Liu, Z. Qin, M. Elkashlan, Z. Ding, A. Nallanathan, and L. Hanzo, "Nonorthogonal Multiple Access for 5G and Beyond," Proceedings of the IEEE. Vol. 105, Issue. 12 , Dec. 2017.

[9] Y. Saito, A. Benjebbour, Y. Kishiyama, and T. Nakamura, "System level performance evaluation of downlink non-orthogonal multiple access (NOMA) Under Various Environments," in Proc. IEEE Annu. Symp. Pers. Indoor Mobile Radio Commun., London, U.K., Sep. 2013, pp. 611-615. 
[10] L. Liu, C. Yuen, Y. L. Guan, and Y. Li, "Capacity-achieving iterative LMMSE detection for MIMO-NOMA systems," in Proc. IEEE Int. Conf. Commun., Kuala Lumpur, Malaysia, May 2016, pp. 1-6.

[11] L. Lei, D. Yuan, C. K. Ho, and S. Sun, "Power and channel allocation for nonorthogonal multiple access in 5G systems: Tractability and computation," IEEE Trans. Wireless Commun., vol. 15, no. 12, pp. 8580-8594, Dec. 2016

[12] F. Fang, H. Zhang, J. Cheng, and V. C. M. Leung, "Energy-efficient resource allocation for downlink non-orthogonal multiple access network," IEEE Trans. Commun., vol. 64, no. 9, pp. 3722-3732, May. 2016.

[13] B. Di, L. Song, and Y. Li, "Sub-channel assignment, power allocation, and user scheduling for non-orthogonal multiple access networks," IEEE Trans. Wireless Commun., vol. 15, no. 11, pp. 7686-7698, Nov. 2016.

[14] Y. Sun, D. W. K. Ng, Z. Ding, and R. Schober, "Optimal joint power and subcarrier allocation for full-duplex multicarrier non-orthogonal multiple access systems," IEEE Trans. Commun., vol. 65, no. 3, pp. 1077-1091, Mar. 2017

[15] J. Choi, "Minimum power multicast beamforming with superposition coding for multiresolution broadcast and application to NOMA systems," IEEE Trans. Commun., vol. 63, no.3, pp. 791-800, Mar. 2015

[16] F. Alavi, K. Cumanan, Z. Ding, and A. G. Burr, "Robust beamforming techniques for non-orthogonal multiple access systems with bounded channel uncertainties," IEEE Commun. Lett., vol. PP, no. 99, pp. 1-1, 2017.

[17] F. Alavi, K. Cumanan, Z. Ding, and A. G. Burr, "Beamforming techniques for nonorthogonal multiple access in 5G cellular networks," IEEE Trans. Veh. Technol., vol. 67, no. 10, pp. 9474-9487, Oct. 2018.

[18] M. F. Hanif, Z. Ding, T. Ratnarajah, and G. K. Karagiannidis, "A minorizationmaximization method for optimizing sum rate in the downlink of non-orthogonal multiple access systems," IEEE Trans. Signal Process., vol. 64, no. 1, pp. 76-88, Jan. 2016

[19] Y. Zhang and M. Guizani, "Game Theory for Wireless Communications and Networking," 1st ed. CRC Press, 2011 pp. 4-20.

[20] A. Tarighat, M. Sadek, and A. H. Sayed. A multiuser beamforming scheme for downlink MIMO channels based on maximizing signal-toleakage ratios," in Proc. IEEE International Conference on Acoustics, Speech, and Signal Processing, vol. 3, Philadelphia, PA, 2005, pp. 1129- 1132.

[21] Mohammed Fadhil, Nor Fadzilah Abdullah, Mahamod Ismail, Rosdiadee Nordin, Cebrail Ciftlikli, Musaab Al-Obaidi, "Maximizing signal to leakage ratios in MIMO $\mathrm{BCH}$ cooperative beamforming scheme', International Journal of Electrical and Computer Engineering (IJECE)Vol.9, No.5, October 2019, ISSN: 2088-8708, pp37013713

[22] Mohammed Fadhil, Nor Fadzilah Abdullah, Mahamod Ismail, Rosdiadee Nordin, , Abduljalil Saif, Musaab Al-Obaidi, 'Power Allocation in Cooperative NOMA MUMIMO Beamforming Based on Maximal SLR Precoding for 5G', Journal of Communications, vol. 14, no. 8, pp. 676-683, 2019.

[23] Ferdi KARA, Hakan KAYA "Spatial Multiple Access (SMA): Enhancing performances of MIMO-NOMA systems," 2019 42nd International Conference on Telecommunications and Signal Processing (TSP)

[24] Kizilirmak, R. Caglar, and H. Khaleghi Bizaki. "Non-orthogonal multiple access (NOMA) for 5G networks." Towards 5G Wireless Networks-A Physical Layer Perspective 83 (2016). 
[25] L. Lei, D. Yuan, C. K. Ho, and S. Sun. Dec. Joint optimization of power and channel allocation with non-orthogonal multiple access for $5 \mathrm{G}$ cellular systems. in Proc. IEEE Global Telecommun,2015. Conf. (GLOBECOM), pp. 1-6.

[26] Y. S. Cho, J. Kim, W. Y. Yang, and C. G. Kang, "MIMO-OFDM wireless communications with MATLAB," John Wiley \& Sons, 2010.

[27] Fan J, Zhang Y (2019) Energy efficiency of massive MU-MIMO with limited antennas in downlink cellular networks. Digit Signal Process 86:1-10

[28] G. H. Golub and C. F. Van Loan, "Matrix Computations," Johns Hopkins University Press Press, 3rd edition.

[29] P. Cheng, M. Tao and W. Zhang, "A new SLNR-based linear precoding for downlink multi-user multi-stream MIMO systems", IEEE Commun. Lett, vol. 14, no. 11, pp. 1008-1010, Nov. 2010

[30] Wen, Yujie, Xiaotian Zhou, Fang Fang, Haixia Zhang, and Dongfeng Yuan. "Nash Bargaining Based Power Allocation and Relay Selection for Cooperative NOMA Aided Spectrum Sharing Systems." In 2020 International Conference on Computing, Networking and Communications (ICNC), pp. 1063-1068. IEEE, 2020

[31] Xie, Xianzhong, et al. Robust power allocation based on game theory for multi-user MIMO system with SLNR precoding. Vehicular Technology Conference IEEE, 2014.

[32] Y. Sun, D. W. K. Ng, Z. Ding, and R. Schober, "Optimal joint power and subcarrier allocation for full-duplex multicarrier non-orthogonal multiple access systems," IEEE Trans. Commun., vol. 65, no. 3, pp. 1077-1091, Mar. 2017

[33] A. Baharlouei and B. Jabbari, "Efficient and Fair Power and Subchannel Allocation in Multiuser OFDM Networks," IEEE Communi. Lett., Vol.17, no.10, Oct.2013.

[34] A. Talukdar, Mobile Computing, 2E. Tata McGraw-Hill Education, 2010.

[35] Falah H. Ali Indu Shakya, "Collaborative spreading for the downlink of overloaded CDMA", January 2009, Wireless Communications and Mobile Computing 10(3):383393

[36] Hoi Yin Shutoy ; Deniz Gunduz ; Elza Erkip ; Yao Wang, "Cooperative Source and Channel Coding for Wireless Multimedia Communications" IEEEE Journal Of Selected Topics In Signal Processing, Vol. 1, No. 2, August 2007.

[37] Afaq, Muhammad, "Performance Analysis of Selected Cooperative Relaying Techniques" Blekinge Institute of Technology, School of Engineering.

[38] K. Higuchi and A. Benjebbour, "Non-orthogonal multiple access (NOMA) with successive interference cancellation for future radio access," IEICE Transactions on Communications, vol. 98, no. 3, pp. 403-414, Mar. 2015.

[39] R. Jain, D. Chiu, and W. Hawe, "A quantitative measure of fairness and discrimination for resource allocation in shared computer systems," Eastern Research Lab, Digital Equipment Corporation, DEC Technical Report 301, Sept. 1984. 